\title{
Quelles améliorations apportées pour améliorer la prise en charge de la douleur dans nos structures d'urgence?
}

\author{
How to improve the management of pain in our emergency departments?
}

\author{
P. Ray \\ (C) SFMU et Lavoisier SAS 2018
}

Cinquante à soixante pour cent des patients expérimentent la douleur en structure d'urgence [1]. Grâce au soutien institutionnel de Mundipharma (Paris, France), ce numéro spécial des Annales françaises de médecine d'urgence permet d'appréhender les actions correctrices à mettre en œuvre pour améliorer la prise en charge des patients douloureux. Avant toute mesure, il est toujours utile de regarder ce que l'on fait. Ainsi, malgré les nombreuses recommandations, de nombreuses études dénoncent un nombre insuffisant de patients soulagés après leur prise en charge en structure d'urgence, $40 \%$ environ. Les freins à l'administration d'antalgiques en urgence sont multiples : le plus classique étant la sous-estimation de l'intensité douloureuse par le personnel soignant ou médical, notamment chez les patients âgés [2], l'absence de son évaluation objective, mais aussi la surcharge du service qui altère la qualité des soins, quel qu'il soit, une insuffisance de formation et une priorisation donnée à l'urgence vitale (insuffisance respiratoire aiguë...). L'ensemble des auteurs sollicités pour ce numéro spécial insiste sur l'importance de la protocolisation pour la prise en charge de la douleur. Cela passe évidemment par l'administration d'un antalgique dès l'infirmière organisatrice d'accueil (IOA), avec dans certains centres l'administration de morphine à libération rapide, mais également par la réévaluation de cette douleur par l'IOA, lorsque le patient attend trop longtemps en salle d'attente, ainsi que la sensibilisation des équipes à la réévaluation de l'intensité de la douleur, mais aussi des médecins, en secteur. Il faudrait probablement considérer (donc analyser, quantifier, suivre) la douleur comme un indicateur qualité (au même titre que le temps de passage...), ce qui permettrait de sensibiliser les équipes et de fédérer celles-ci autour d'objectifs communs. Une autre

\section{P. Ray $(\triangle)$}

SAMU-SMUR-Urgences Hôpital François-Mitterand,

5, boulevard Jeanne d'Arc, 21000 Dijon, France

e-mail : patrick.ray@chu-dijon.fr, patrick.ray@tnn.aphp.fr

Université de Bourgogne, Faculté de médecine de Dijon

7, boulevard Jeanne d'Arc, 21079 Dijon Cedex, France façon est de mettre en place des protocoles, en particulier infirmiers, évidemment en accord avec les recommandations locales et/ou des sociétés savantes [3] et validés par les médecins (qui ne doivent pas désinvestir la douleur) et l'institution, permettant une certaine liberté d'administration des antalgiques par les IDE. Enfin, il est probablement nécessaire d'innover pour s'améliorer. Cela passe par des innovations galéniques que commercialisent les industriels avec de nouvelles voies d'administration qui seraient plus rapides en efficacité et/ou avec moins d'effets secondaires potentiels, et/ou avec une potentialisation positive avec d'autres médicaments déjà existants. Mais s'améliorer veut également dire changer notre regard vis-à-vis de la douleur pour tendre vers un nouveau paradigme, un peu galvaudé, qui deviendrait : « time is... », comme nous l'avons intégré pour l'infarctus du myocarde il y a maintenant 20 ans, pour l'accident vasculaire cérébral ou pour le choc septique plus récemment, surtout lorsque l'on connaît les conséquences physiologiques néfastes d'une oligoanalgésie dans beaucoup de situations urgentes. Il nous reste donc encore beaucoup à travailler pour améliorer la prise en charge de la douleur en structures d'urgence.

\section{Références}

1. Boccard E, Adnet F, Gueugniaud PY, et al (2011) Pain management in adult patients in emergency care units in France in 2010. Ann Fr Med Urgence 1:312-9

2. Ricard-Hibon A, Duchateau FX, Vivien B (2012) Out-of-hospital management of elderly patients for trauma injury. Ann Fr Anesth Reanim 31:e7-e10

3. Vivien B, Adnet F, Bounes V, et al (2011) Recommandations formalisées d'experts 2010 : sédation et analgésie en structure d'urgence (réactualisation de la conférence d'experts de la SFAR de 1999). Ann Fr Med Urgence 1:57-71 